Preprint

UCRL-JC-148223

\title{
Development of Large- Aperture, Light-Weight Fresnel Lenses for Gossamer Space Telescopes
}

S. Dixit, R. Hyde, A. Weisberg, J. Early, M. Rushford, J. Britten

This article was submitted to The $3^{\text {rd }}$ American Institute of Aeronautics and Astronautics Gossamer Spacecraft Forum, Denver, U.S. Department of Energy CO, April 22-25, 2002

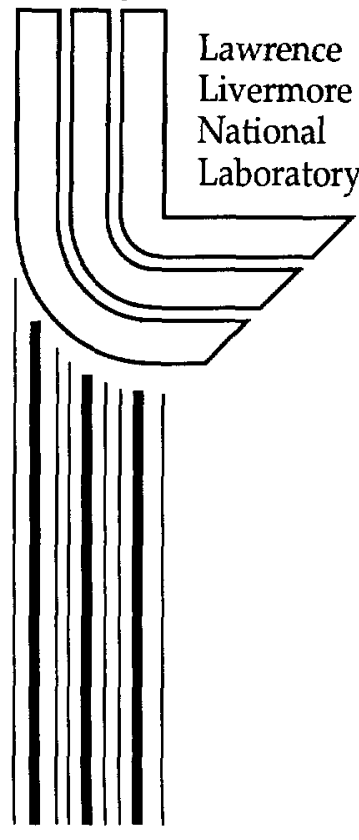

Lawrence

Livermore

National

April 29, 2002 


\section{DISCLAIMER}

This document was prepared as an account of work sponsored by an agency of the United States Government. Neither the United States Government nor the University of California nor any of their employees, makes any warranty, express or implied, or assumes any legal liability or responsibility for the accuracy, completeness, or usefulness of any information, apparatus, product, or process disclosed, or represents that its use would not infringe privately owned rights. Reference herein to any specific commercial product, process, or service by trade name, trademark, manufacturer, or otherwise, does not necessarily constitute or imply its endorsement, recommendation, or favoring by the United States Government or the University of California. The views and opinions of authors expressed herein do not necessarily state or reflect those of the United States Government or the University of California, and shall not be used for advertising or product endorsement purposes.

This is a preprint of a paper intended for publication in a journal or proceedings. Since changes may be made before publication, this preprint is made available with the understanding that it will not be cited or reproduced without the permission of the author.

This work was performed under the auspices of the United States Department of Energy by the University of California, Lawrence Livermore National Laboratory under contract No. W-7405-Eng-48.

This report has been reproduced directly from the best available copy.

Available electronically at http://www.doc.gov/bridge

Available for a processing fee to U.S. Department of Energy

And its contractors in paper from

U.S. Department of Energy

Office of Scientific and Technical Information

P.O. Box 62

Oak Ridge, TN 37831-0062

Telephone: (865) 576-8401

Facsimile: (865) $576-5728$

E-mail: reports@adonis.osti.gov

Available for the sale to the public from

U.S. Department of Commerce

National Technical Information Service

5285 Port Royal Road

Springfield, VA 22161

Telephone: (800) 553-6847

Facsimile: (703) 605-6900

E-mail: orders@ntis.fedworld.gov

Online ordering: http://www.ntis.gov/ordering.htm

\section{OR}

Lawrence Livermore National Laboratory

Technical Information Department's Digital Library

http://www.llnl.gov/tid/Library.html 


\title{
Development of large-aperture, light-weight Fresnel lenses for Gossamer space telescopes
}

\author{
Sham Dixit, Rod Hyde, Andrew Weisberg, Jim Early, \\ Mike Rushford and Jerry Britten \\ Lawrence Livermore National Laboratory, Livermore, California 94550
}

\section{Introduction}

In order to examine more distant astronomical objects, with higher resolution, future space telescopes require objectives with significantly larger aperture than presently available. NASA has identified a progression in size from the $2.4 \mathrm{~m}$ aperture objective currently used in the HUBBLE space telescope[1,2], to $25 \mathrm{~m}$ and greater in order to observe, e.g., extra-solar planets. Since weight is a crucial factor for any object sent into space, the relative weight of large optics over a given area must be reduced[3]. The areal mass density of the primary mirror for the Hubble space telescope is $\sim 200 \mathrm{~kg} / \mathrm{m}^{2}$. This is expected to be reduced to around $15 \mathrm{~kg} / \mathrm{m}^{2}$ for the successor to Hubble - the next generation space telescope (NGST)[4]. For future very large aperture telescopes needed for extra-solar planet detection, the areal mass density must be reduced even further. For example, the areal mass density goal for the Gossamer space telescopes is $<1 \mathrm{~kg} / \mathrm{m}^{2}$. The production of lightweight focusing optics at $>10 \mathrm{~m}$ size is also an enabling technology for many other applications such as Earth observation, power beaming, and optical communications.

One future space telescope project, Eyeglass[5], has proposed the use of a $>25 \mathrm{~m}$ diffractive (Fresnel) lens on a thin membrane. The advantages of a diffractive lens over conventional refractive and reflective optics are many-fold. Compared to refractive lenses, the volume of transparent material that is actually required to focus light in a Fresnel lens is very small, with the rest of the material being there simply as support. In fact, a $25 \mathrm{~m}$ filled-aperture Fresnel lens for visible light requires only $\sim 0.6 \mathrm{~kg}$ of glass to operate! This allows for Fresnel lenses to be made in thin membranes independent of the F-number of the optics. Long F-number Fresnel lenses also offer substantial advantages over equivalent reflective devices in their tolerance to surface-figure errors and hence the precision of the mounting structure. Conventional reflectors require Angstrom-level control of the surface position, which is why large mirrors are thick. In comparison, long F-number Fresnel lenses can tolerate cm-scale ripples perpendicular to the membrane without significant focal spot distortion, as long as the parallelism of the membrane is good. This increased tolerance to surface ripples for transmissive optic comes about because light reflecting off a surface bump picks up a path delay equal to twice the ripple height where as light going through a bump picks up almost no path delay as the path delay introduced by one surface is nearly completely cancelled by the second surface. This is schematically illustrated in figure 1. This increased surface ripple tolerance makes 
Fresnel lenses ideal for use on a thin, continuous, membrane where the precision along its plane can be controlled much easier than perpendicular to it.
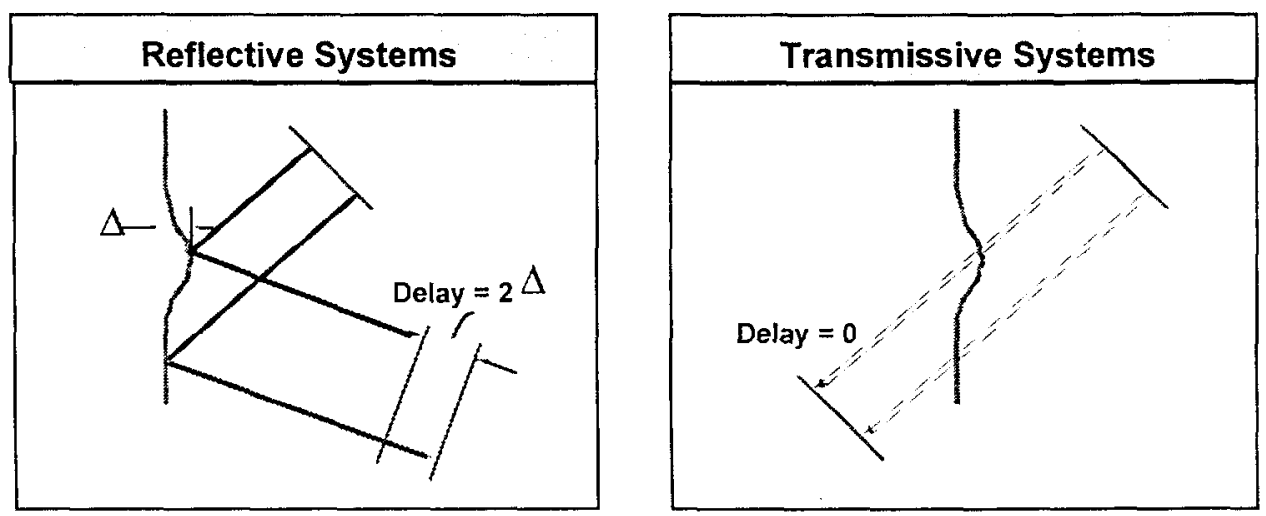

Figure 1. Effect of a surface ripple in reflective vs. transmissive systems

One disadvantage of Fresnel lenses is their highly chromatic operation. Normally, different wavelengths are focused by the lens at different points, causing a multi-spectral image to become distorted. However, by inserting an inverse Fresnel lens into the collecting optics, this chromaticity can be corrected, allowing for broadband operation [5]. This feature has been theoretically before. The theoretical calculations have also shown that by using deeper surface-relief structures, even greater spectral coverage can be achieved [5]. Experimental demonstration has used a complicated layout of holographic diffractive lenses [6,7].

In this paper we discuss our development of large aperture Fresnel lenses for use in future space telescopes such as the Gossamer. Fabrication of a monolithic 25-m class Fresnel lenses is beyond the scope of any available technology today. Therefore we are investigating a segmented approach to the Fresnel lens fabrication. As a first step in this development effort, we fabricated monolithic Fresnel lenses and verified that they can be used in color-corrected diffractive telescopes. The following section describes the results of this work. In section 3 we describe the fabrication and the optical testing of a $75-\mathrm{cm}$ diameter, segmented Fresnel lens. This is to demonstrate that high quality segmented lenses can be fabricated. Section 4 then summarizes our current effort to scale up this technology to fabricate a 5-m diameter Fresnel lens.

\section{Demonstration of color corrected diffractive telescopes}

An Eyeglass space telescope consists of two distinct spacecraft, separated in space by approximately 1 kilometer [5]. The telescope's large aperture is provided by a transmissive diffractive lens, acting as a Magnifying Glass. This large lens gathers and gently focuses light, directing it to a distant focal surface, where it is collected by a separate, much smaller, space telescope serving as the Eyeglass's mobile Eyepiece. As a first step in advancing this concept for space applications, we wanted to demonstrate the feasibility of wide band operation of such long F-number, diffractive telescopes. For this, we built a diffractive telescope based upon a $20 \mathrm{~cm}, \mathrm{f} / 100$ lens. The lens was fabricated 
by a lithographic process, generating a 4-level, digitally blazed, phase profile [8]. In order to chromatically correct this diffractive lens, we combined it with a $4 \mathrm{~cm}$ relay lens (a refractive achromat) and a $2.2 \mathrm{~cm}, \mathrm{f} / 11$, inverse diffractive lens.

This experiment successfully demonstrated our ability both to chromatically correct diffractive telescopes, and to do so with diffraction-limited performance. In figure 2 below we show the telescope's point spread function (PSF) for $633 \mathrm{~nm}$ laser light and for broad-band (470-700 $\mathrm{nm}$ ) white-light. The broadband light is clearly focused into a tight spot, which has the predicted $95 \mu \mathrm{m}$ Airy width. We also used this telescope (piping in light via turning mirrors) to look at real astronomical objects, taking pictures of the Moon, Saturn, Jupiter, and the Sun. These are shown in figure 3.

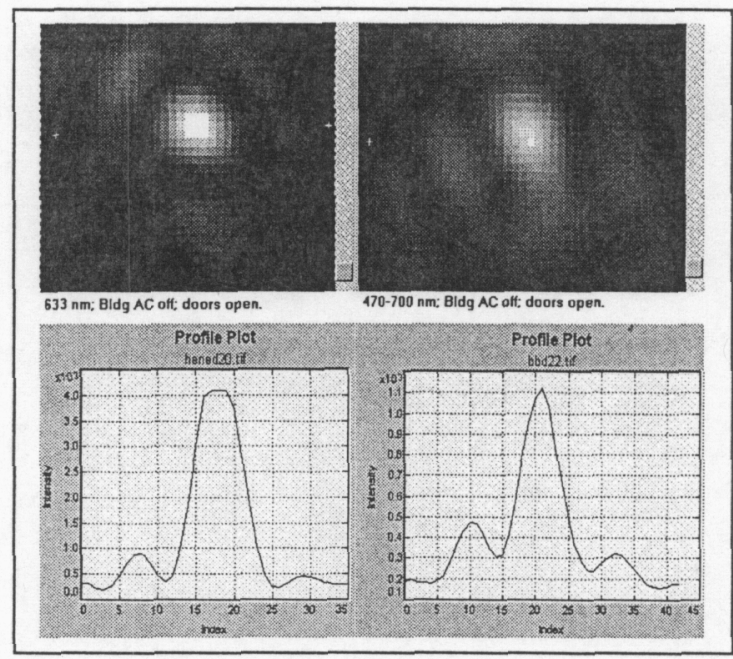

Figure 2. Observed focal spots for a $20-\mathrm{cm}$ aperture, $20 \mathrm{~m}$ focal length diffractive telescope. The left panels are for $633 \mathrm{~nm}$ $\mathrm{HeNe}$ laser while the right panels for illumination with a broad-band $(470-700 \mathrm{~nm})$

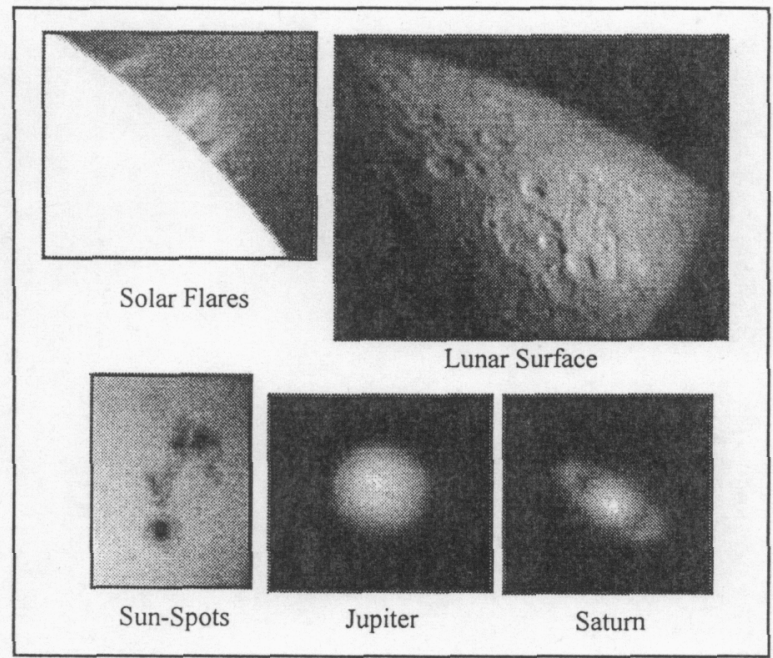

Figure 3.Images of various astronomical objects captured using the $20-\mathrm{cm}$ aperture diffractive telescope

Next, we used our existing photolithographic tools to make a $50 \mathrm{~cm}$ diameter, $\mathrm{f} / 100$, 4-level diffractive lens in a fused silica substrate [8]. To our knowledge, this is the largest, optically precise, phase-profile diffractive lens in existence. We used this silica lens as the primary in a $50 \mathrm{~cm}$ aperture, $\mathrm{f} / 100$, color-corrected, diffractive telescope. The large diameter and slow f/number of this lens, leads to a $>50 \mathrm{~m}$ long design for the telescope. We used the NOVA laser bay provide the large, vibrationally and environmentally controlled, location needed for fielding this diffractive telescope. The optical design was basically a scaled-up version of the one used for the $20 \mathrm{~cm}$ telescope. Figure 4 below shows line-outs of the focused spots achieved with incident plane waves, one using a $543 \mathrm{~nm}$ laser, and the other composed of broadband white light (470 to 700 $\mathrm{nm})$. Both plane waves are tightly focused to spots of the diffraction-limited size, 62 microns, i.e., 9 pixels for our CCD.

These 20- and 50-cm telescope demonstrations clearly validate the concept of a fully color corrected diffractive telescope. 


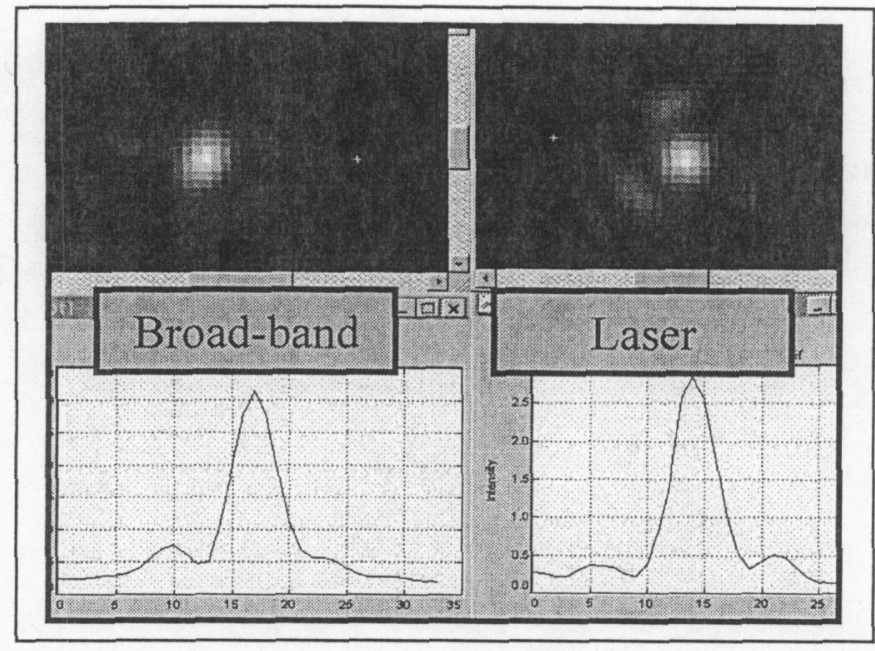

Figure 4. Point spread functions for the $50-\mathrm{cm}$ diffractive telescope. Again monochromatic demonstration was using $633 \mathrm{~nm}$ laser while the broad-band corresponds to $470-700 \mathrm{~nm}$ illumination

\section{Fabrication of a segmented Fresnel lens}

The scale up of the diffractive telescopes to larger apertures and also to light-weight materials raises many new challenges. First of all the lens substrate material has to be space suitable and has to be amenable to the fabrication of the Fresnel lens structure on its surface. We have examined plastics and glasses. Although some versions of polyimides show promise in terms of their space suitability, considerable technology development is required for their use as a large area Fresnel lens substrate. Thin glass sheets up to 1-m size are being fabricated routinely for the flat-panel industry. Although many of these glass materials undergo structural changes when exposed to ultraviolet radiation, the UV degradation can be circumvented by mixing in additional UV absorbers during the production of these sheets. Glasses have the limitation that they are inherently brittle and folding and launching a monolithic, large aperture lens is impractical with currently available launch platforms. A way to get around this problem is to fabricate a Fresnel lens that consists of segments that are precision assembled. Such a segmented lens can then be folded into a smaller volume for launch and 'opened up' when deployed in space. We have developed this concept further using Origami folding concepts which help minimize overlapping folds. Figure 5 below shows one such design of a segmented lens and how it can be folded up into a hat-box like structure.

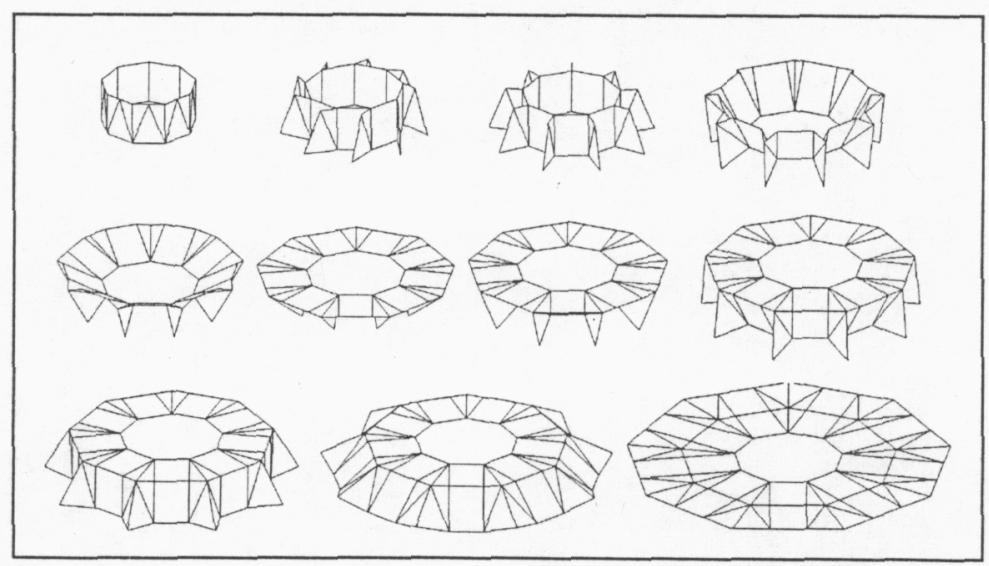

Figure 5. Schematic of the folding and the unfolding sequence of a 5-m Fresnel lens 
Based on these technological and economic considerations, we have adopted a segmented approach to scale up the Fresnel lens technology to larger sizes. Rather than building a full-sized, monolithic lens, we will instead create the overall lens by forming, and then joining together, a number of smaller lens segments. The virtue of this approach is that it splits an extremely difficult task into two, much more tractable, parts production of the lens panels and their precision assembly into a large lens. We are developing technologies in both these areas. These are discussed in section 4 .

Before, embarking on the segmented lens development for large apertures, we wanted to demonstrate that the segmented lens can focus light into a small enough focal spot to be useful in a telescope. Even more importantly, we wanted to verify that the lens performs well after its folded and unfolded. Our demonstration lens was a designed to have a $75 \mathrm{~cm}$ optical aperture ( $80 \mathrm{~cm}$ physical diameter) and a focal length, at $532 \mathrm{~nm}$, of 39 meters at $532 \mathrm{~nm}$. This represents an f-number of 52, and requires an outermost Fresnel zone-width of $55 \mu \mathrm{m}$. The lens consisted of 6 segments, as shown below. This particular Origami configuration was chosen in order to avoid nested folds.
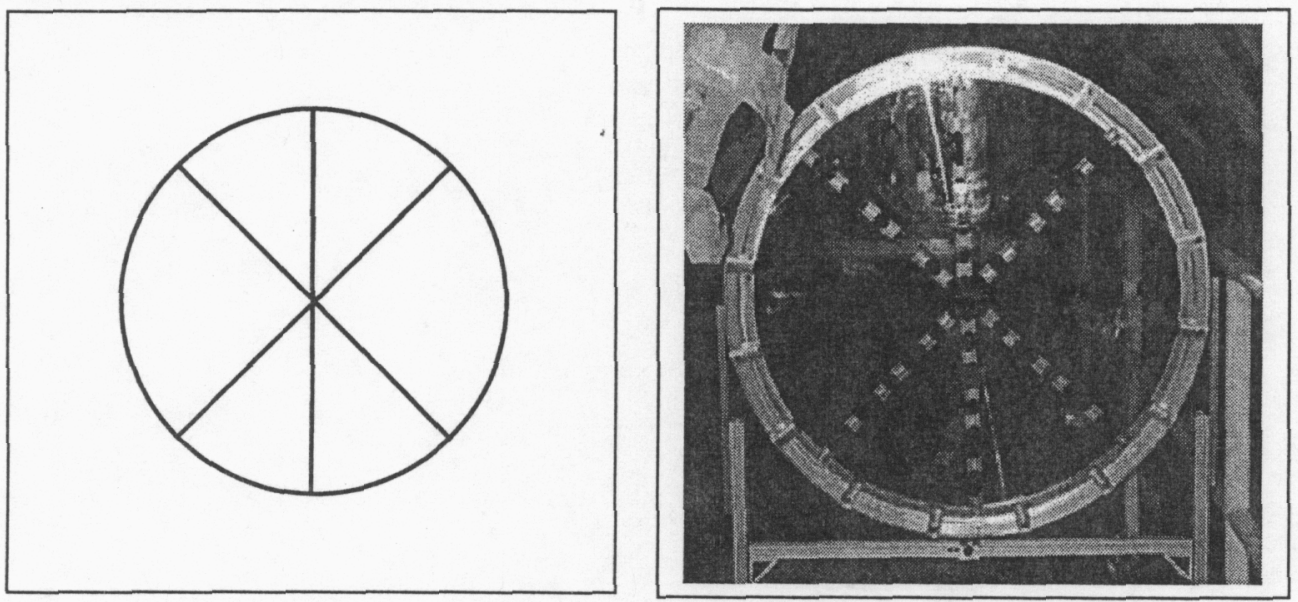

Figure 6. A schematic (left) and the actual picture of the $75-\mathrm{cm}$ diameter segmented Fresnel lens

The fused silica panels for this lens were produced using a traditional grind and polish technique. In order to maintain a common absolute thickness and a constant wedge across the full aperture, the lens panels were produced together, by polishing an $80 \mathrm{~cm}$ circular disk, and then separating it into 4 quadrant-segments. We used a two-mask lithography process to apply a 4-level diffractive lens profile to their surfaces. Two of the quadrants were then cut in half, leaving us with six finished lens panels.

In order to assemble our segmented lens, we used precisely positioned fiducials on each of the panels to accurately align them relative to each other, and then seam-together the six lens panels. The fiducials were printed during the photolithography step and, as such, are tightly liked to the Fresnel lens pattern. The seams were composed of 1 inch wide, 1.5 mil Ti-alloy films glued to the glass panels using a UV curable polymer adhesive. The assembled lens is also shown in figure 5.

Optical testing of the lens consisted of examining the focal spot characteristics when illuminated by a monochromatic plane wave. We used a $75 \mathrm{~cm}$ aspherical lens to provide 
a $532 \mathrm{~nm}$ plane-wave. Given this plane-wave and beam-path, the segmented diffractive should, if successful, deliver a tight $(\sim 60 \mu \mathrm{m})$ spot at a 39 meter focal distance. The figure 6 above shows the focal spot produced by this segmented lens. The PSF measurement shows that this segmented lens does optically perform as a full-aperture lens, delivering a tight, diffraction-limited, focal spot. The lens patterning, panel alignment, and inter-panel seaming are accurate enough to deliver a high-quality optical lens.

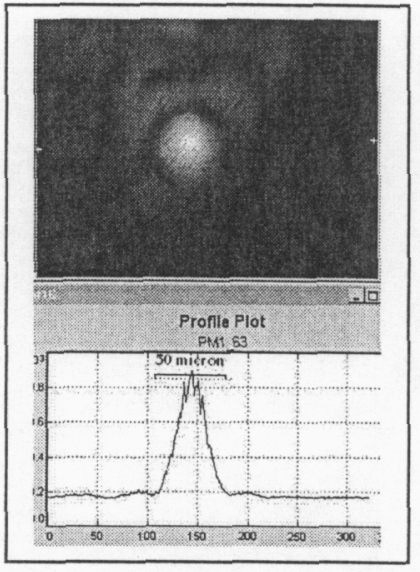

Figure 7 (left). Focal spot produced by the seamed lens before folding

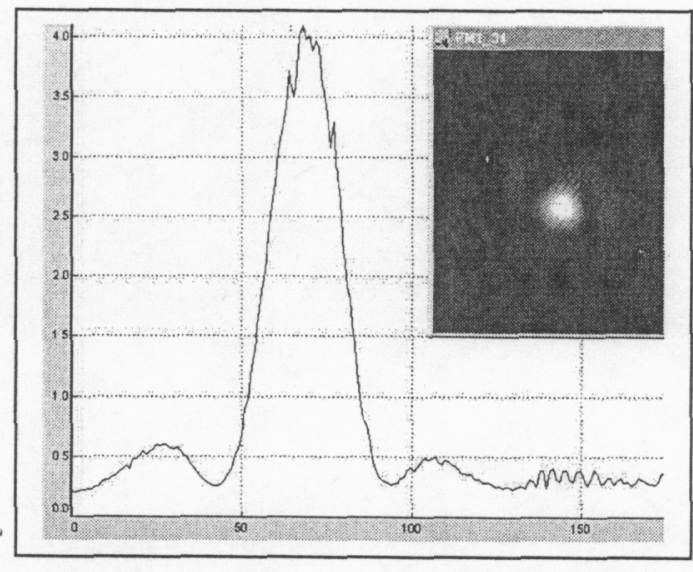

Figure 9 (right). Focal spot produced by the seamed lens after folding and unfolding

After measuring the as-built performance of the lens, and demonstrating that this segmented lens performs similarly to a monolithic lens, we then concentrated on folding the lens. We removed the lens from the mount, folded it, unfolded it and placed it back in the mount. Care was taken so as to not cause the thin seaming tabs to crease; thus insuring that the folded seams did settle into the desired max-bend-radius configuration. The photographs in the figure below show some of the steps during the folding/unfolding process.
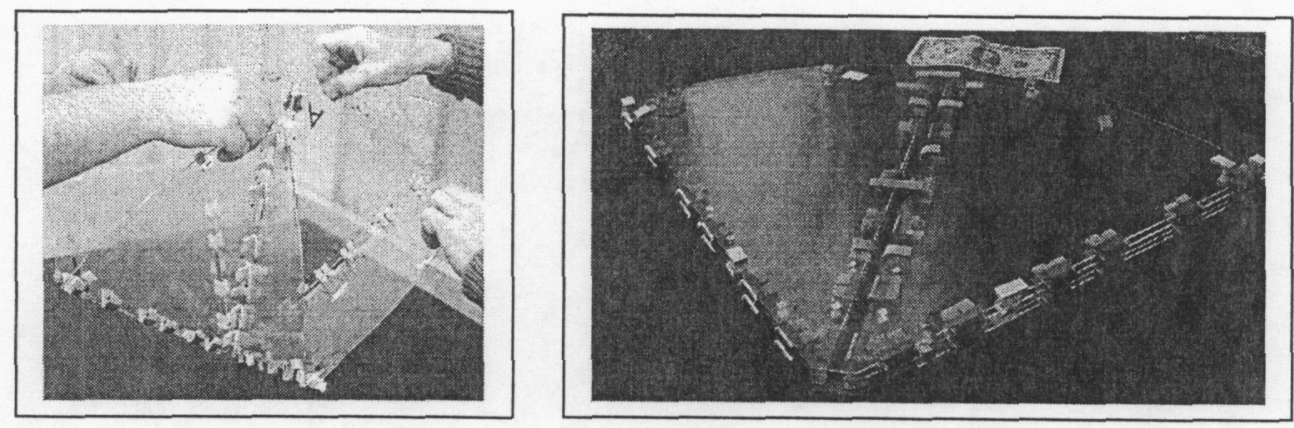

Figure 8. Folding and unfolding of the 6segmented seamed Fresnel lens

After the lens was folded-up and then unfolded again, we reinserted it into the optical mount and remeasured its focal spot. Figure 9 shows the lens's post-folding PSF. The focal spot is very similar in size to that observed before the folding and unfolding operation. These test results clearly show that the lens performs very well, the same after 
folding/unfolding as it did beforehand. This demonstration successfully validates the multi-panel approach we're proposing for producing large aperture Fresnel lenses.

\section{Development of the 5-meter Fresnel lens}

With successful demonstration of the color corrected diffractive telescopes and the demonstration of the segmented Fresnel lens, we are now embarking on scaling this concept to even larger sizes. Our current effort is focused on building and testing a 5-m diameter high quality Fresnel lens. When complete, this will be the largest diffractive lens ever built. The 5-m Fresnel lens design we have chosen (shown in fig 9) has an Origami like pattern to enable it to be folded after its assembly for transportation and possibly a launch test. This lens design consists of a total of 81 pieces made up of 18 rectangles, 36 right angled triangles and 27 isosceles triangles. The table below lists the sizes of these shapes.

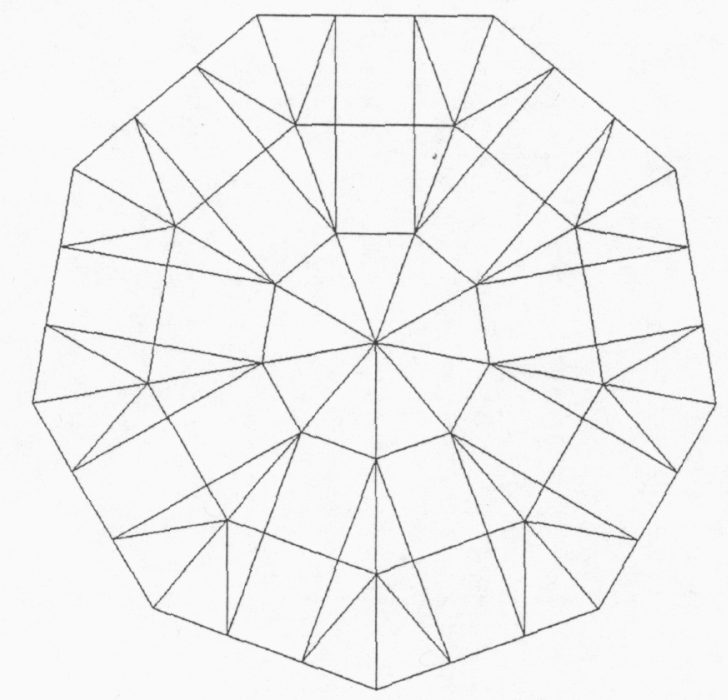

Figre 10. A schematic design of a 5-m Origami based Fresnel lens

\begin{tabular}{|l|l|l|}
\hline & Number & Size \\
\hline Rectangles & 18 & $567 \times 780 \mathrm{~mm}$ \\
\hline Right angle triangles & 36 & $283 \times 780 \mathrm{~mm}$ \\
\hline Isosceles triangles & 27 (incl 9 for the center) & 567 (base) $\times 760$ (height) $\mathrm{mm}$ \\
\hline
\end{tabular}

The high degree of symmetry is chosen in order to minimize the different types of tooling necessary for handling during the patterning and assembly steps. While fused silica would be the material of choice, fabricating large thin sheets of fused silica is prohibitively expensive. There are derivatives of the borosilicate glasses that are being manufactured in large volumes for the flat panel displays. Properties of these glasses tend to mimic, with some penalties, those of silica. For instance, glass does not offer quite the broad-spectral transparency of silica, has 5-10 times higher thermal expansion, and presents some (probably minor) radiation concerns. However, because it can be processed at much lower temperatures, glass is generally cheaper and easier to acquire than silica. So, while silica would be our ideal material choice, we have settled on the borosilicate glasses as the baseline material for our 5-m Fresnel lens. We have identified 
several candidates that appear promising for Eyeglass applications. Schott produces thin float glass, Borofloat, in meter sizes and thickness down to $700 \mu \mathrm{m}$. Corning produces meter-sized sheets of 1737 and 7059 glasses by a fusion process in thickness as low as $500 \mu \mathrm{m}$. Down draw processes are used by Corning (0211 \& 0214 glass) and by Schott (D263T \& AF45) to produce much thinner (although smaller) glass micro-sheets. Both companies produce sheets about $35-40 \mathrm{~cm}$ wide; Coring goes down to $75 \mu \mathrm{m}$ thickness and Schott to $30 \mu \mathrm{m}$. These sheets are all borosilicate glasses. As such they deliver silicalike transmission over the visible regime, suffering extra absorption primarily in the UV. Their greatest drawbacks compared to silica are UV-induced browning under long-term exposures, and substantially higher thermal expansion $\left(4-7 \times 10^{-6} /{ }^{\circ} \mathrm{C}\right.$ compared to $5 \times 10^{-7} /{ }^{\circ} \mathrm{C}$ ). The former problem can be eliminated by addition of Cerium (as is done with the Corning $0214 \mathrm{mix}$ ), while the higher thermal expansion coefficients are probably still acceptable.

Next we measured the transmission wavefront of the $700 \mu \mathrm{m}$ thick, meter size Corning 1737 and Schott Borofloat using phase shifting interferometry. The typical wavefront of a Corning 1737 sheet is shown in figure 11 along with horizontal and vertical slices at several locations on this sample. The wavefront over a large area $(\sim 740$ x $800 \mathrm{~mm}$ ) was measured in four overlapping 440 x $440 \mathrm{~mm}$ segments and the resulting wavefronts were 'stitched' together to calculate the transmission wavefront of the full part. We see that this glass exhibits predominantly $\sim 1$-d thickness variations with $\sim 100$ $\mathrm{mm}$ scale lengths. The peak-to-valley thickness is of the order of $10 \mu \mathrm{m}$ and as such is too large for these sheets to be useable as is to fabricate a Fresnel lens. The wavefront aberrations on the glass would severely degrade the focal spot quality. The Borofloat wavefronts showed much more shorter scale thickness variations and therefore we chose the Corning 1737 as the baseline material for forming the Eyeglass lens panels.
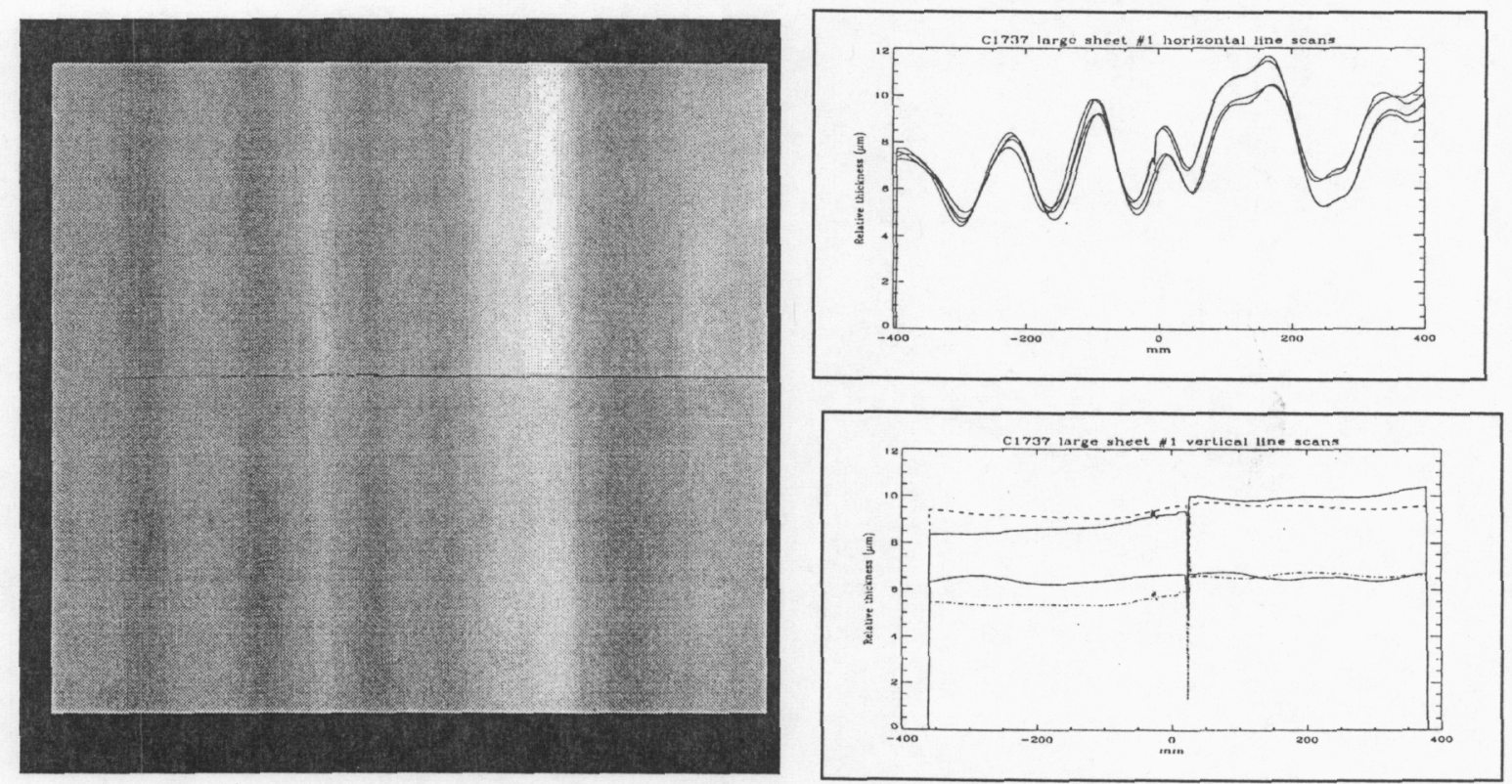

Figure 11. Transmitted wavefront of a Corning 1737 thin glass sheet. The image extent is approximately $700 \times 800 \mathrm{~mm}$. Horizontal (top) and vertical (bottom) scans at several locations on this image are shown on the right. 
Since the Eyeglass lens panels are required to have transmission wavefronts of the order of $\lambda / 10$, a further flattening of the thickness of the glass sheets is needed. Conventional finishing techniques like grind and polish are not suitable for flattening thin glass sheets. Moreover, non-destructive techniques such as ion milling are prohibitively expensive for large areas. The most promising approach was a spatially controlled etching method developed at LLNL [9]. This makes use of numerically controlled, chemically-assisted, small-tool etching. As noted above, the ripples in our glass microsheets are predominantly 1-D. This spatial variation was exploited by the development of a 1-D micro-etcher. This tool featured a 1-D etcher in which dilute HF acid was delivered through a slot, formed an etchant-line on the glass sheet, and was then actively removed (dewetted) via the Marangoni effect. The location of this etch-head over the glass was controllable with a 1-D translation-stage, and was moved about so that local dwell-time could be used to selectively etch more-or-less glass at given locations. This etch process was actively controlled using feedback from a real-time thickness-measuring interferometer. In figure 12 below, we show a flat plateau etched into a rippled glass micro-sheet. The original (largely 1-D) ripples were smoothed down from their initial, 12 $\mu \mathrm{m}$, depths to a flat plateau having $\sim 100 \mathrm{~nm}$ residual variation in about 10 hours of processing. We are currently extending the size capability of this tool to $\sim 1 \mathrm{~m}$ panels and are also building fully 2 -d tools for flattening the glass wavefronts.

\section{Etched 4" x 5" Plateau}

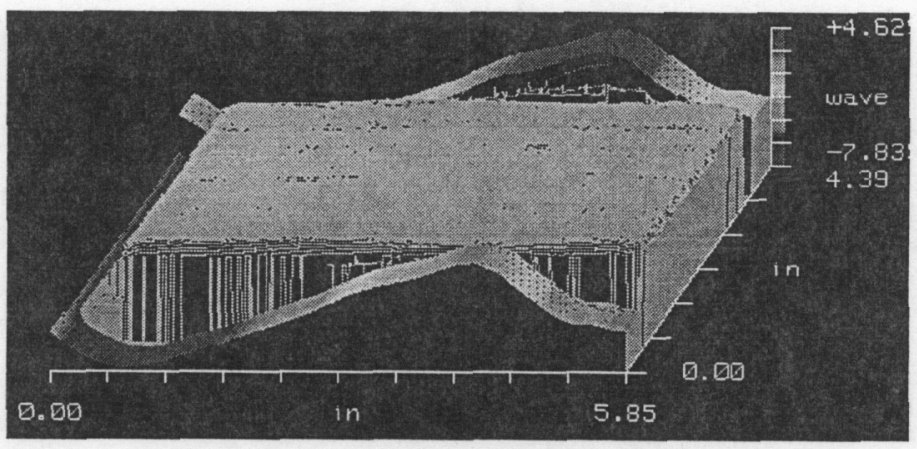

Figure 12. demonstration of flattening of thin glass sheets by the wet-etch figuring technique

The remaining process needed to form the lens panels is the application of the Fresnel lens diffractive surface profile. We plan to use photolithography to do the patterning. LLNL has extensive (world-leading) expertise in this operation and as such we do not foresee any technical issues in patterning the individual panels for the 5-m Fresnel lens.

Once the panels have been patterned and cut to the desired shape, they have to be precision assembled into a 5-m Fresnel lens. As was done in the 75-cm folding lens case, we will use a fiducial-based technique for aligning lens panels. In order to achieve $\pm \lambda / 10$ wavefront quality, we need $\pm 6 \mu \mathrm{m}$ radial positioning accuracy over the entire 5 meter aperture. While the absolute accuracy required for our 5 meter lens is the same as that of the $75 \mathrm{~cm}$ one, the relative accuracy is, of course, about 6.5 times greater. Achieving this large improvement in relative alignment accuracy, i.e., attaining $6 \mu \mathrm{m}$ precision over a 5 
meter span instead of a $75 \mathrm{~cm}$ one, constitutes a major challenge to the assembly of our 5 meter lens.

We believe the largest source or error in precision assembly is likely to be caused by thermal expansion. The glass panels have thermal expansion coefficients of about 4 $\mathrm{ppm} /{ }^{\circ} \mathrm{C}$, so $\sim 1{ }^{\circ} \mathrm{C}$ temperature excursions can be ruinous. In order to assure a highprecision lens, our assembly station must incorporate rigorous temperature control, maintaining thermal uniformity over the 5 meter assembly to $\sim 0.1{ }^{\circ} \mathrm{C}$. This kind of thermal control also dictates that external thermal sources be kept to a minimum during the assembly process. We are currently constructing a semi-automated, machine-based assembly station that has good thermal isolation and temperature control. Machine-based assembly will allow us to deal with the large size and number of parts, to effectively access the large assembly span, while maintaining a thermally controlled environment.

A schematic of the assembly station is shown in the figure below. The lens is assembled on the upper surface of a thermally controlled, vibrationally-isolated worktable. Thermal control is achieved by placing the table in an insulated enclosure, and by incorporating an actively controlled heat exchanger just under the surface of the table. The glass panels are placed, aligned, and seamed on the table by use of several specialized tools, mounted as hands on a movable arm which traverses the working surface. One hand is used to place the panels into the vicinity (1-2 mil accuracy) of their proper location. A second hand is then used to perform local metrology along the interface with an, already attached, neighbor panel. After digesting the local metrology data, this hand then shifts the new panel into its proper precise location and joins it to its neighbors. A third hand is used for post-assembly verification measurements by traversing the lens, measuring fiducial locations.
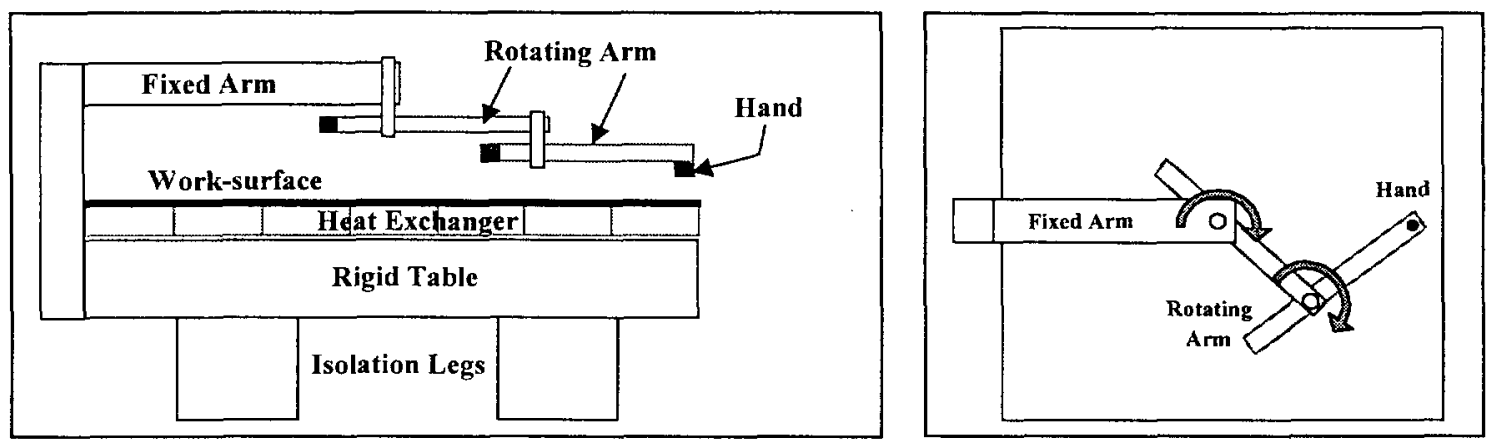

Figure 13. A schematic diagram of the 5-m Fresnel lens assembly station layout

After assembling the 5 meter lens, we will actively characterize and verify its alignment, before releasing it for optical testing. This metrology consists of measuring the global locations of the alignment fiducials over the entire lens. The location of the fiducials combined together with the rigidity of the glass panels and the thermal stability of the environment would enable us to verify the overall lens quality. 
While these component-level tests will be valuable, and verify that the lens has been successfully built, a far more complete and compelling approach is an optical testing of the assembled 5-m lens; this directly demonstrates the full-up global precision of the Fresnel lens. The large aperture and the long focal length of the pose extreme challenges for this testing. A collimated-beam to focus test requires that one generate a 5-m aperture collimated beam. This also requires a $>250 \mathrm{~m}$ aberration free beam path for testing. A point to point imaging test requires a minimum path length of $4 \mathrm{f} \mathrm{i.} \mathrm{e.} 1 \mathrm{~km}$. A way around this is to test the lens at a higher order focus. For example if we tested the lens at $5^{\text {th }}$ order focus or used 5 times the wavelength $(2.5 \mu \mathrm{m})$ the required $4 \mathrm{f}$ distance reduces by a factor of 5 . We are examining these issues and will report on the progress and results in the future.

This research was performed under the auspices of the U. S. Department of Energy by Lawrence Livermore National Laboratory under contract W-7405-Eng-48. The authors acknowledge excellent contributions from Curly Hoaglan, Leslie Summers, John Toeppen and lan Thomas. 
References:

[1] L. L. Endelman, 'The Hubble Space Telescope mission, history, and systems,' (SPIE High-Speed Photography and Photonics: Cambridge, 1990.) Proc. SPIE 1358 422-441 (1991), B. Garfield and J. Rendell Eds.

[2] L. L. Endelman, 'Hubble Space Telescope: now and then,' (SPIE High-Speed Photography and Photonics: Santa Fe, 1996.) Proc. SPIE 2869 44-57 (1997), D. L. Paisley, and A. M. Frank, Eds.

[3] J. R. P. Angel, 'Glass mirrors for space telescopes,' (SPIE Proceedings of the Optical Fabrication and Testing Workshop: Large Telescope Optics, Albuquerque, 1985.) Proc. SPIE 542, 32-34 (1985), R. S. McDowell Ed. .

[4] H. S. Stockman, M. Stiavelli, J. C. Mather, 'The NGST science mission,' (Astron. Soc. Pacific Science with the NGST: Greenbelt, 1997.) Astron. Soc. Pacific Conference Series 133, 24-33 (1998).

[5] R. A. Hyde, 'Eyeglass. 1. Very large aperture diffractive telescopes,' Appl. Opt. 38 (19), 4198-4212 (1998).

[6] D. Faklis and G. M. Morris, 'Broadband imaging with holographic lenses,' Opt. Eng. 28 592-598 (1989).

[7] R. E. Hufnagel, 'Achromatic holographic optical system,' U.S. patent 4550973 (5 November 1985)

[8] I. M. Barton, J. A. Britten, S. N. Dixit, L. J. Summers, I. M. Thomas, M. C. Rushford, K. Lu, R. A. Hyde and M. D. Perry, 'Fabrication of large-aperture, light weight diffractive lenses for use in space,' Appl. Opt. 40 447-451 (2001).

[9] M. C. Rushford, J. A. Britten, C. R. Hoaglan, I. M . Thomas, L. J. Summers and S. N. Dixit,'Wet-etch figuring: Optical surfacing by controlled application of etchant solution using the Marangoni effect,' Proceedings SPIE 4451249 - 258 (2001). 